\title{
Production d'énergie à partir de forêt tropicale humide : une étude de cas en Guyane française
}

FrançOIS PINTA ${ }^{1}$

PHILIPPE GIRARD ${ }^{2}$
1 Cirad
Unité de recherche Biomasse-énergie TA B-42/16
73, rue Jean-François Breton
34398 Montpellier Cedex 5
France
2 Institut international d'ingénierie de l'eau et de l'environnement 01 BP 594, Ouagadougou 01
Burkina Faso

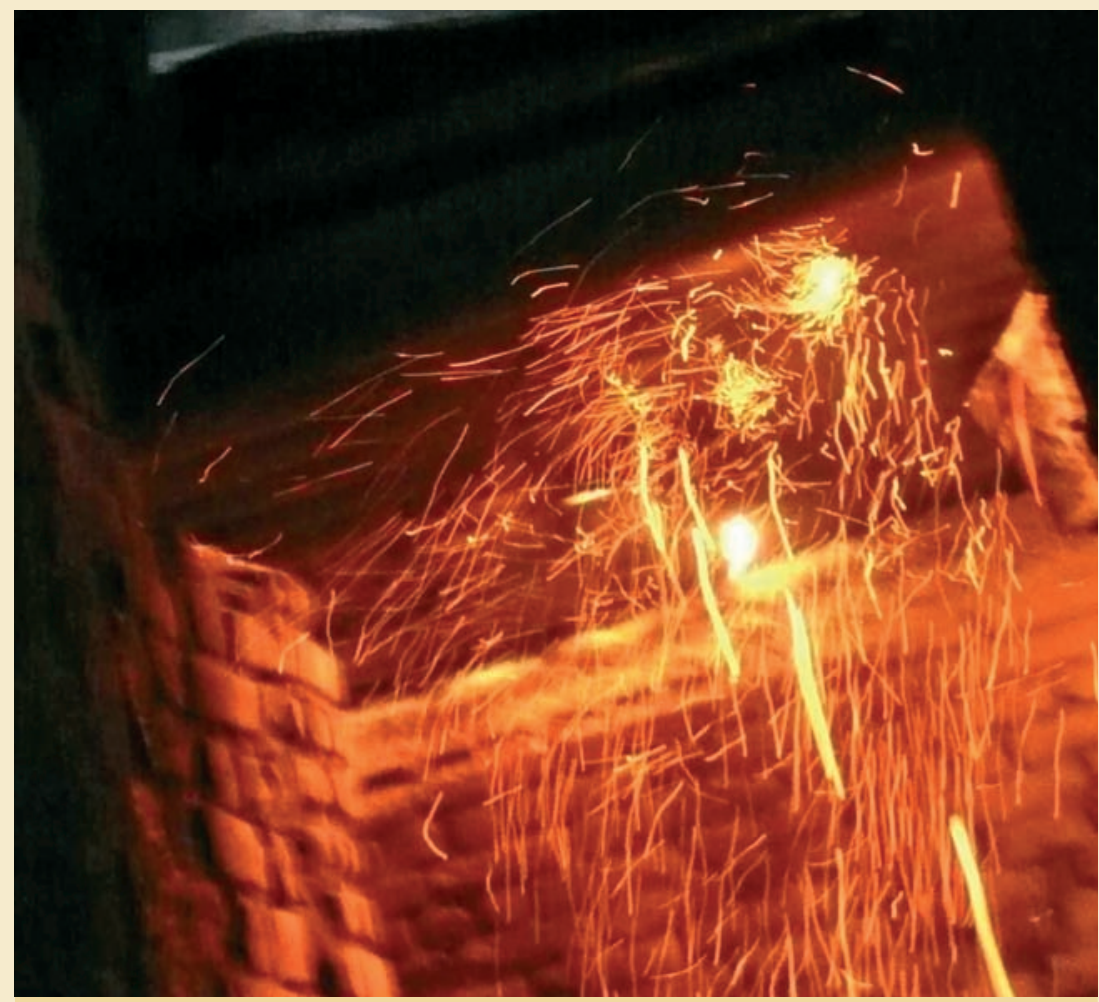




\section{RÉSUMÉ}

\section{PRODUCTION D'ÉNERGIE À PARTIR DE FORÊT TROPICALE HUMIDE : UNE ÉTUDE DE CAS EN GUYANE FRANÇAISE}

L'un des verrous à la mise en œuvre de projet de génération d'électricité à partir de la biomasse réside dans la bonne appréciation de la nature de la ressource et de sa disponibilité. Cet article s'inspire de travaux qui ont été réalisés en 2007 afin d'apprécier la faisabilité de la mise en place de centrales dendro-électriques de vingt mégawatts en Guyane française. L'analyse de la disponibilité en ressources de biomasse a été au cœur de l'étude. L'originalité de l'approche consiste à apprécier la capacité de la forêt à fournir de manière durable tout ou partie des volumes nécessaires et sans remettre en cause la gestion actuelle. Différentes ressources ont été étudiées dont la valorisation de bois issus de deux gisements : l'exploitation forestière pour la production de bois énergie et l'exploitation mixte de bois énergie en complément de l'exploitation de bois d'œuvre. Des scénarios ont été étudiés pour la zone côtière où se concentre la majorité de la demande électrique. Les travaux ont montré une disponibilité importante permettant d'approvisionner des centrales électriques de cinq et dix mégawatts de capacité électrique. L'étude de préfaisabilité d'une centrale de cinq mégawatts électriques près du village de Régina a montré la faisabilité économique du projet utilisant du bois issu de l'exploitation mixte des forêts de production et du bois récupéré sur l'emprise des pistes forestières. Cependant, la validation des schémas prévisionnels d'exploitation durable de bois énergie en forêt naturelle aménagée exige des recherches spécifiques complémentaires.

Mots-clés : biomasse, bois énergie, électricité, aménagement forestier, micro-économique.

\section{ABSTRACT}

\section{ENERGY PRODUCTION FROM HUMID TROPICAL FORESTS: A CASE STUDY IN FRENCH GUIANA}

One of the main obstacles to projects for power generation from biomass is clearly related to the accuracy of assessments of the nature and availability of the resource. This article draws on work performed in 2007 to assess the feasibility of setting up a wood-fired power plant in French Guiana. The main part of the study consists of an analysis of the availability of biomass resources for $20 \mathrm{MW}$ power plants. The initial approach was to assess the capacity of the forest to support sustainable supplies of all or part of the volume required, without a major overhaul of current management. Different biomass resources were studied, including fuelwood from sustainable natural forest use only and fuelwood from forest use in combination with timber harvesting. Several scenarios were studied for the coastal area, where the majority of electricity demand is concentrated. The study showed significant availability capable of supplying several 5 and $10 \mathrm{MW}$ power plants. The prefeasibility study for a $5 \mathrm{MW}$ power plant project near the village of Régina demonstrated the economic feasibility of combining the use of fuelwood from production forests and waste wood from logging track creation and maintenance. However, further specific research is needed to confirm the provisional plan for sustainable fuelwood production from natural rain forests.

Keywords: biomass resources, fuelwood, power generation, forest management, micro-economic.

\section{RESUMEN}

\section{PRODUCCIÓN DE ENERGÍA EN BOSQUES TROPICALES HÚMEDOS: ESTUDIO DE CASO EN LA GUAYANA FRANCESA}

Una de las trabas para llevar a cabo un proyecto de generación de electricidad a partir de la biomasa estriba en la buena apreciación de la naturaleza del recurso y de su disponibilidad. Este artículo se inspira en los trabajos realizados en 2007 para evaluar la viabilidad del establecimiento de centrales dendroeléctricas de veinte megavatios en la Guayana Francesa. El estudio se centró principalmente en el análisis de la disponibilidad de recursos de biomasa. La originalidad del enfoque consiste en estimar la capacidad del bosque guayanés para suministrar toda o una parte de los volúmenes necesarios de modo sostenible y sin cuestionar el actual manejo. Se estudiaron distintos recursos, entre los que se encuentra el aprovechamiento de leña procedente de dos fuentes: la explotación forestal dedicada a la producción de leña y la explotación de leña como complemento del aprovechamiento de madera de construcción en una explotación mixta. Se estudiaron varios escenarios en la zona costera, en la que se concentra la mayoría de la demanda eléctrica. Los estudios mostraron una importante disponibilidad, capaz de abastecer centrales eléctricas de cinco y diez megavatios. El estudio de previabilidad para la implantación de una central de cinco megavatios eléctricos cerca del pueblo de Regina mostró la viabilidad económica del proyecto. El suministro de leña vendría de la explotación mixta de bosques de producción y de la recuperación en la apertura de pistas forestales. No obstante, para validar los esquemas de pronóstico de aprovechamiento sostenible de leña en un bosque natural manejado, se deben realizar investigaciones específicas complementarias.

Palabras clave: biomasa, leña, electricidad, manejo forestal, microeconómico. 


\section{Introduction}

L'approvisionnement en énergie des pays tropicaux constitue un verrou important au développement économique des pays non producteurs de pétrole. Pour lever ce verrou, diverses options sont envisagées par les pouvoirs publics et les bailleurs de fonds internationaux. Nombre d'entre elles privilégient les énergies renouvelables et tout particulièrement le recours à la biomasse.

Si les plantations énergétiques ont souvent été envisagées et mises en œuvre, comme c'est le cas de la sidérurgie brésilienne ou des biocarburants à l'échelle internationale, par contre, l'aménagement de forêts tropicales humides à vocation énergétique constitue un domaine sensible encore peu exploré. Seules quelques études ont été menées sur la valorisation des déchets d'exploitation forestière, pour lesquels les résultats se sont révélés décevants sur le plan économique et risqués sur le plan environnemental. Toutefois, cette approche a progressé plus rapidement dans les zones de forêts sèches, traditionnellement exploitées à des fins de bois de feu, où l'aménagement s'avère être la seule option possible pour en garantir la pérennité (BERTRAND, MONTAGNe, 2009 ; ICHAOU, ROULETTE, 2006).

La question de l'exploitation durable des forêts tropicales humides à des fins énergétiques a été abordée en Guyane française par des équipes de l'Onf ${ }^{1}$ et du Cirad ${ }^{2}$. Il s'agissait d'évaluer la faisabilité technico-économique de l'approvisionnement en bois combustible de centrales électriques d'une capacité totale de vingt mégawatts pour le réseau $\mathrm{Edf}^{3}$. Pour cela, le besoin annuel en biomasse brute, d'humidité moyenne de quarante pour cent (\%) sur brut (FALCH, PINTA, 2007), a été évalué à deux cent soixante-dix mille tonnes.

Cet article présente la disponibilité en biomasse forestière exploitable pour des usages énergétiques, étudie les modalités de sa mobilisation et évalue la faisabilité économique de cette filière. II traite, d'une part, d'une exploitation forestière mixte de production de bois énergie en complément du bois d'œuvre et, d'autre part, d'une exploitation forestière durable à vocation énergétique.

\section{Méthodologie}

L'étude a consisté à définir, localiser et caractériser quantitativement et qualitativement les gisements de biomasse. Les gisements ont été identifiés à partir des données des services forestiers et sur la base des recherches sur la dynamique forestière menées par le Cirad (GouRLET-FLEURY et al., 2005). Les gisements correspondant au bois mobilisable dans les zones à vocation agricole ont été localisés à partir des données de la Direction de l'agriculture et des forêts qui pilote le schéma de développement agricole de la Guyane française. En outre, les inventaires forestiers réalisés dans les années 1970 et les dispositifs d'étude des peuplements mis en place dans les années 1980 permettent une estimation du volume de bois disponible, de la variabilité spatiale et de la qualité moyenne des produits.

L'exploitation forestière n'étant possible que sur des pentes inférieures à $40 \%$ et hors des zones marécageuses, les zones ne présentant pas ces caractéristiques ont été exclues des zones exploitables.

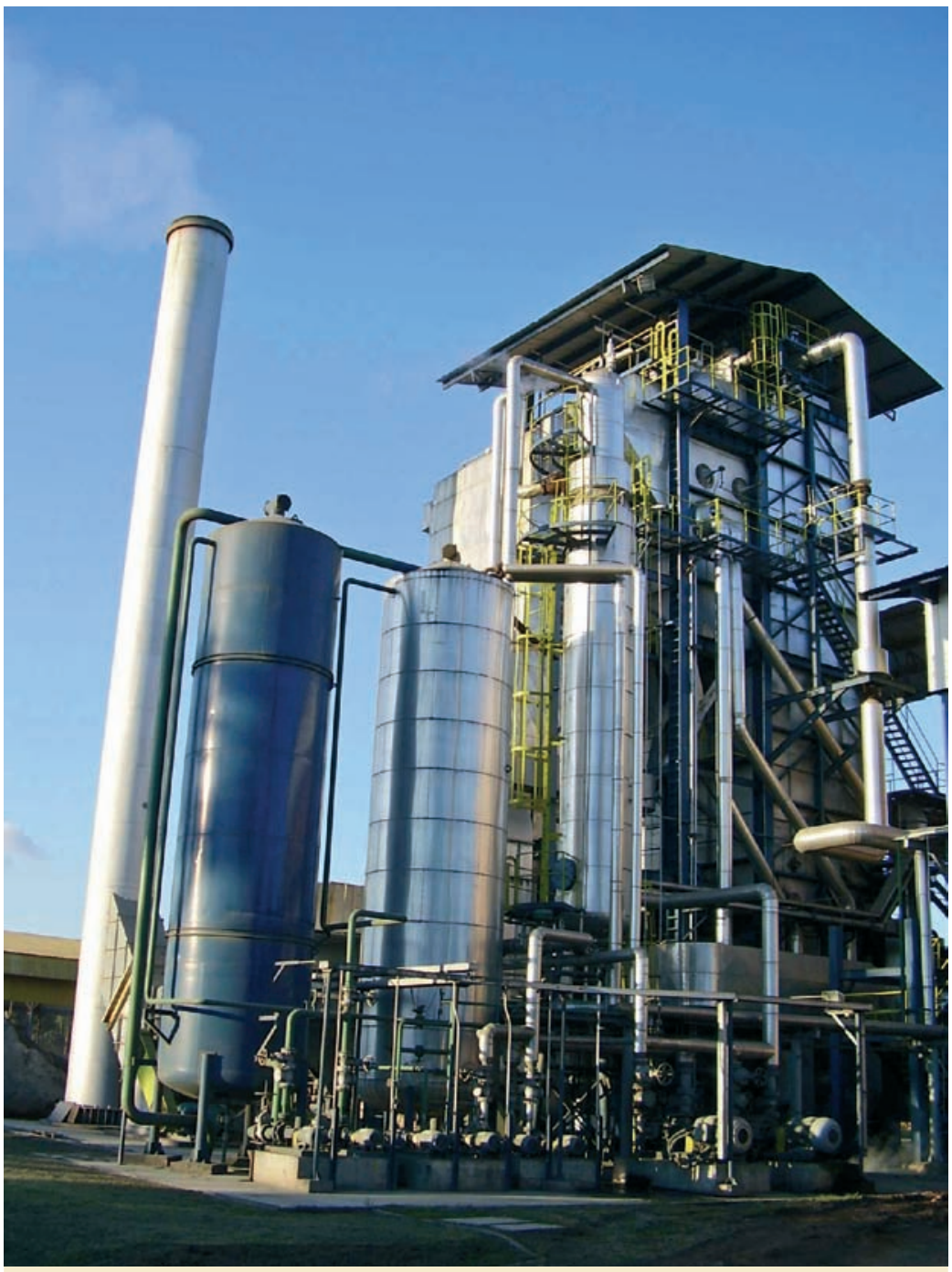

Photo 2.

Exemple de centrale à bois de 10 mégawatts électriques au Brésil. Photo F. Pinta.

${ }^{1}$ Office national des forêts, entreprise nationale de gestion et aménagement des forêts.

2 Centre de coopération internationale en recherche agronomique pour le développement.

3 Électricité de France, entreprise nationale de distribution de l'énergie électrique. 


\section{La disponibilité en biomasse} des houppiers et des petites tiges de diamètre inférieur à vingt centimètres $(\varnothing<20 \mathrm{~cm})$ nécessite un façonnage contraignant, ce qui diminue la productivité du chantier d'exploitation forestière. Pour des raisons économiques, ce type de biomasse a donc été écarté de la ressource considérée comme mobilisable.

À l'inverse, l'exploitation des arbres de grande taille $(\varnothing>80 \mathrm{~cm})$ est retenue. Ces classes de diamètres représentent 7 à $8 \%$ du volume de bois mobilisable 4 ; elles correspondent à des bois habituellement exploités par la filière bois d'œuvre. Le volume unitaire élevé laisse présager une bonne productivité lors de l'exploitation forestière et du transport. Leur conditionnement en plaquettes forestières nécessite une opération spécifique de refente avant broyage. Cette classe d'arbres correspond à des individus matures devant tôt ou tard laisser leur place pour la régénération de la forêt.

Les itinéraires techniques de mobilisation de la biomasse ont été définis à partir des données de terrain et sur la base d'une consultation locale des professionnels forestiers et entrepreneurs de travaux publics et forestiers.

À partir de localisations rationnelles des centrales (proximité du réseau électrique), une analyse de préfaisabilité a été conduite afin d'évaluer les coûts de production de l'électricité (PINTA et al., 2007).

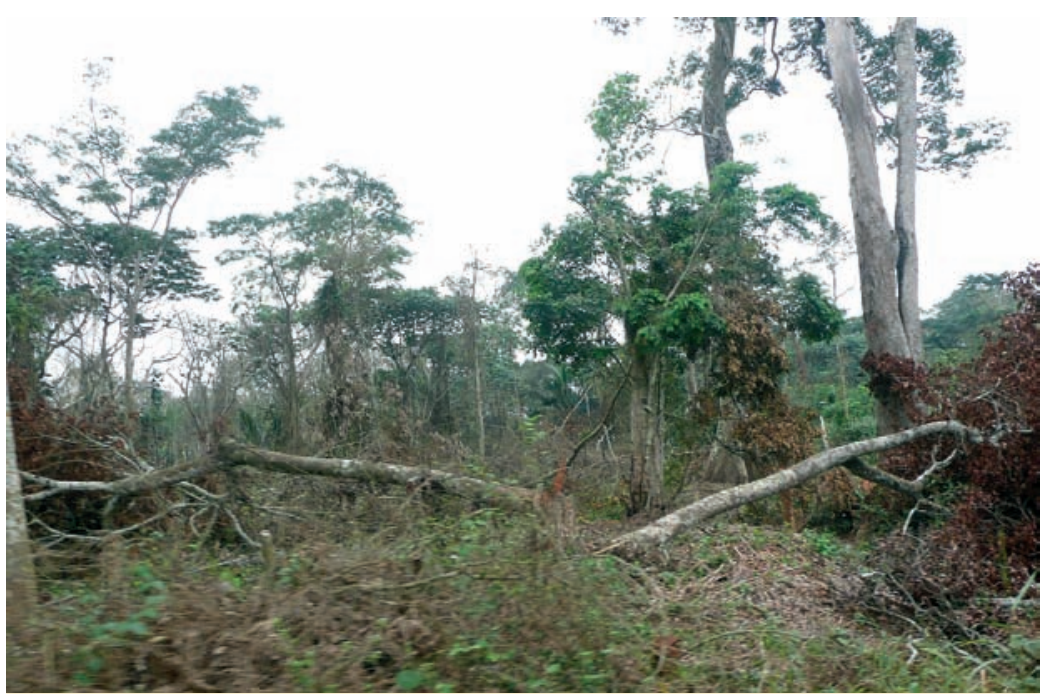

Photo 3.

Abattage d'arbres pour l'énergie en forêt secondaire tropicale. Photo F. Pinta.

\section{Une abondante biodiversité}

Hormis une dizaine de milliers d'hectares de peuplements secondaires et de plantations pour le bois de trituration disséminés sur la frange côtière, deux types forestiers peuvent être distingués en fonction de leur degré d'anthropisation. D'une part, les peuplements primaires qui n'ont jamais été exploités si ce n'est de façon très extensive par les populations amérindiennes. Ils représentent environ sept millions d'hectares, correspondant à la majorité de la surface forestière guyanaise. D'autre part, les peuplements perturbés qui correspondent aux zones touchées par l'exploitation forestière et sont principalement localisées sur la bande côtière (anciens permis forestiers, forêts aménagées) et à proximité des anciens centres de peuplement (placers miniers importants, pénitenciers, villages...). Leur surface est de l'ordre du million d'hectares. Seuls ces deux types forestiers sont donc pris en compte dans le cadre de cette étude.

${ }^{4}$ Le « volume de bois mobilisable » est défini ici comme la fraction des tiges et branches dont le diamètre est supérieur ou égal à $20 \mathrm{~cm}$.
Le nombre d'espèces d'arbres en Guyane française est estimé à mille deux cents, dont environ trois cents espèces de gros arbres accédant à la canopée. La richesse à l'hectare peut culminer à plus de deux cents espèces. Un corollaire à cette diversité est l'absence d'espèces franchement dominantes et la grande variabilité des produits exploitables. Si dix essences forestières représentent à elles seules $52 \%$ du volume et $57 \%$ de la biomasse en moyenne dans les forêts de terre ferme, il faut plus de quarante-cinq essences forestières pour atteindre $80 \%$ du volume en zone marécageuse.

Les espèces guyanaises se caractérisent par une densité anhydre moyenne très élevée : autour de 0,73 (soit 0,83 à $12 \%$ d'humidité). Cette caractéristique est assez bien connue avec une base de données fournie pour toutes les essences exploitées. Les connaissances sont beaucoup plus modestes en ce qui concerne le taux de cendres et de silice, qui est inconnu pour plus de $40 \%$ des essences mais dont l'importance est essentielle pour les choix d'équipements en aval (broyage, grilles de chaudière...). Les données disponibles sont peu fiables compte tenu de l'extrême variabilité spécifique et de la délicatesse des procédures d'échantillonnage et d'analyse. Par exemple, pour l'angélique (Dicorynia guianensis), les valeurs de taux de silice varient de $0,03 \%$ à $1,43 \%$.

Au sein des peuplements perturbés, l'exploitation forestière passée et actuelle se caractérise par de faibles prélèvements (de l'ordre de deux à trois tiges par hectare exploité) concentrés sur les tiges de gros diamètre $(\varnothing>70 \mathrm{~cm})$ d'une trentaine d'essences. Trois essences représentent à elles seules près des trois quarts du volume prélevé. À ce prélèvement, il convient d'ajouter les 


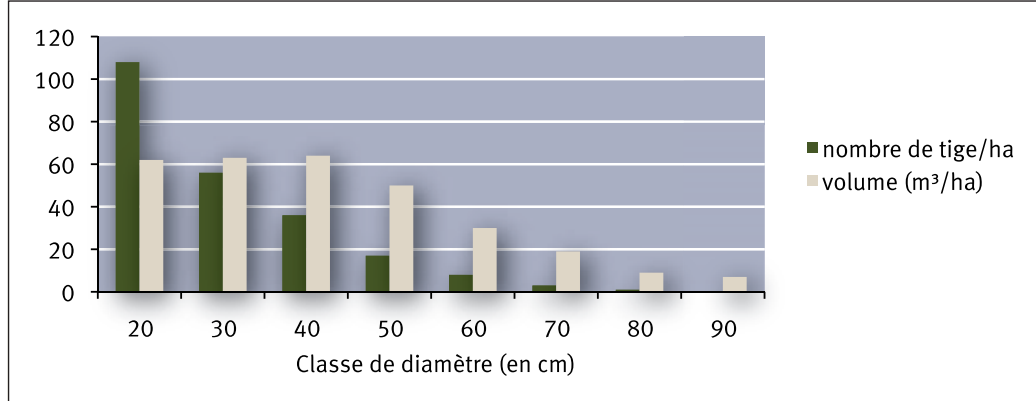

Figure 1.

Structure diamétrique et volumes de bois mobilisable par classe de diamètre.

pertes dues aux dégâts d'exploitation, qui restent toutefois limitées par rapport au volume de bois sur pied total. En ce qui concerne le volume total de biomasse disponible, ce gisement est donc semblable au précédent, comme le montrent les résultats obtenus sur le dispositif de Paracou (DemEnois et al., 2003).

\section{Une structure irrégulière et une quantité de biomasse variable}

Toutes essences confondues, la structure du peuplement est de type exponentiel décroissant, caractéristique des peuplements irréguliers, dominée par les tiges de petit diamètre (figure 1 ). Il en résulte un volume sur pied de l'ordre de $320 \mathrm{~m}^{3} / \mathrm{ha}$, avec en majorité des tiges de faible dimension (1,3 $\mathrm{m}^{3}$ en moyenne pour les tiges de diamètre supérieur à $20 \mathrm{~cm}$ ).

Cette structure diamétrique est un paramètre important pour orienter le choix du matériel pour la production de plaquettes, car les diamètres extrêmes impliquent des coûts de mobilisation plus élevés que les bois de dimension moyenne. Les tiges de diamètre inférieur à $40 \mathrm{~cm}$, qui représentent plus de $45 \%$ du volume total, abaissent la rentabilité des opérations d'exploitation (notamment au débardage) ou imposent des engins bien spécifiques tels que des porteurs forestiers qui ne sont pas utilisés en Guyane française. Quant aux gros diamètres (supérieurs à $80 \mathrm{~cm}$ ), ils représentent 7 à $8 \%$ du volume de grumes total et doivent être fendus avant broyage.

Par ailleurs, une forte variabilité des peuplements est observée selon le type de drainage du sol et la localisation biogéographique (tableau I).

Un volume moyen de bois mobilisable de $290 \mathrm{~m}^{3} /$ ha en terre ferme pour une biomasse totale (houppier inclus) de 350 t/ha (matière sèche) a donc été retenu comme base d'analyse.

Enfin, les paramètres de taille du bois exploitable et ceux de topographie ${ }^{5}$ conduisent à une réfaction du volume bois de $10 \%$, le volume moyen disponible maximum pour une valorisation du bois énergie étant ainsi évalué à $260 \mathrm{~m}^{3} / \mathrm{ha}$.

\section{Origine des gisements et volumes de biomasse exploitable}

\section{Biomasse issue d'une exploitation forestière mixte (bois d'œuvre et bois énergie)}

La production annuelle de grumes en Guyane française est de l'ordre de $65000 \mathrm{~m}^{3}$. L'exploitation forestière durable est réalisée dans des forêts aménagées d'environ un million d'hectares, incluant des zones de conservation. Le rythme de mise en production est d'environ 12000 ha par an.

L'exploitation du bois d'œuvre repose sur une récolte sélective de deux à trois tiges par hectare en moyenne, réalisée selon la technique dite conventionnelle, qui s'accompagne d'une réduction de la surface terrière 6 du peuplement de $14 \%$ liée à l'abattage et aux dégâts de débardage. À cet impact direct vient s'ajouter la mortalité des tiges abîmées lors de l'exploitation forestière, qui représente 6 à $9 \%$ de la surface terrière trois à cinq ans après l'année d'exploitation.

\footnotetext{
5 Pente maximale de $40 \%$ en zone exploitée.

${ }^{6}$ Indicateur de suivi forestier qui est la surface cumulée des sections des arbres mesurées à 1,30 m de hauteur pour un hectare de forêt (en $\mathrm{m}^{2} / \mathrm{ha}$ ).
}

Tableau I.

Volume de biomasse disponible par type de peuplement (source : Onf Guyane).
Type de peuplement de forêt primaire

Peuplement de terre ferme

Peuplement de zone marécageuse
Densité tiges à l'hectare $(\varnothing>20 \mathrm{~cm})$

180 à 275

120 à 250
Volume bois mobilisable moyen $\left(\mathrm{m}^{3} / \mathrm{ha}\right)$

230 à 370

175 à 340
Biomasse totale sèche* $(t / h a)$
* bois mobilisable + houppier et petites tiges supérieures à $10 \mathrm{~cm}$. 


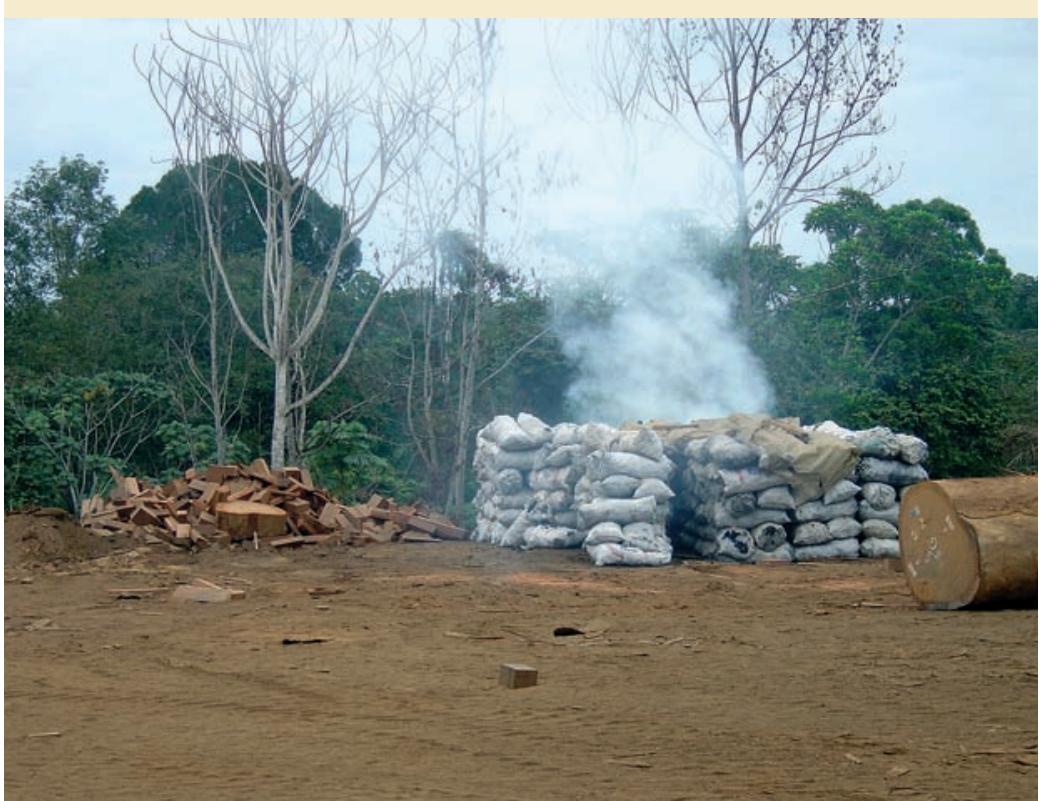

Photo 4.

Production de charbon de bois à partir des déchets dans une scierie au Gabon. Photo F. Pinta.

Le gisement de bois énergie qu'il est possible d'extraire des parcelles exploitées pour le bois d'œuvre peut ainsi provenir de deux sources : la valorisation des bois endommagés et l'exploitation de tiges supplémentaires en éclaircie. Actuellement, l'estimation du volume détruit restant en forêt après exploitation est de $228500 \mathrm{~m}^{3}$ par an, soit de l'ordre de $43 \mathrm{~m}^{3}$ par hectare exploité, dont plus de $80 \%$ du peuplement reste sur pied et vivace après exploitation.

Une exploitation mixte présente donc plusieurs intérêts :

- Récupérer une partie des pertes de matière en forêt pour environ $2 \mathrm{~m}^{3}$ par hectare exploité, dont $1 \mathrm{~m}^{3}$ en récupérant des purges qui pourraient être faites sur parc à grumes et valorisées en bois énergie et $1 \mathrm{~m}^{3}$ supplémentaire par l'accroissement des abattages d'arbres que les exploitants de bois d'œuvre jugent de « qualité limite ». En principe, la qualité de ces grumes devrait permettre que 30 à $50 \%$ du volume soit valorisé comme bois d'œuvre, le reste étant valorisé en bois énergie.
- Récupérer une partie des bois détruits par l'exploitation pour $22 \mathrm{~m}^{3}$ par hectare exploité : les tiges représentent un potentiel d'environ $11 \mathrm{~m}^{3}$ par hectare (soit six tiges). Les essences forestières regroupées sous le nom de " gaulettes » ne sont pas prises en compte car il s'agit de bois trop dur pour être facilement valorisé. Les tiges très endommagées susceptibles de mourir à brève échéance (trois à cinq ans) représentent le même potentiel (GUITET et al., 2009). - Mobiliser 15 à 17 m³/ha de bois supplémentaire en éclaircie, uniquement près des pistes de débardage existantes pour limiter les coûts de mobilisation et les impacts consécutifs à l'ouverture de nouvelles emprises. La mobilisation sans dépasser le seuil maximum de prélèvement durable estimé à un tiers de la surface terrière (GOURLET-FLEURY et al., 2005), tout en considérant l'augmentation espérée du prélèvement de bois d'œuvre à quatre tiges et demie par hectare, ce qui correspondra à une réduction de la surface terrière d'environ $23 \%$. Le prélèvement de six tiges représentant une quinzaine de mètres cubes complémentaires permettrait de ne pas complémentaire serait ainsi réalisée terrière. Le niveau de prélèvement par hectare ne devra pas être trop élevé pour assurer la durabilité de l'exploitation et la qualité du peuplement (composition en essences, état sanitaire). En effet, une ouverture trop importante du peuplement peut le déstabiliser et défavoriser la régénération des espèces commercialement valorisables.

Ainsi, environ $40 \mathrm{~m}^{3}$ par hectare seraient mobilisables en bois énergie sur ces parcelles. Si l'on considère une stabilité de l'activité de la filière, la surface exploitée annuellement représente un potentiel de $240000 \mathrm{~m}^{3}$. Le Programme régional de mise en valeur forestière (ANONYME, 2007) prévoit une dynamisation de la filière, en lien avec l'accroissement démographique et les besoins en construction. En tablant sur un prélèvement de bois d'œuvre de $40 \mathrm{~m}^{3} /$ ha, le volume total escomptable serait ainsi de $320000 \mathrm{~m}^{3}$ par an.

Le principal intérêt de cette exploitation mixte est d'améliorer la gestion durable en dynamisant des peuplements secondaires par une opération d'éclaircie au profit des arbres d'avenir. L'éloignement des forêts de production du bois d'œuvre est un handicap de poids car la distance moyenne de transport est aujourd'hui de cent quarante kilomètres, pistes et routes comprises.

\section{Biomasse issue d'une exploitation exclusivement à vocation énergétique}

Deux types de scénarios sont envisageables :

- exploitation intensive abaissant le volume moyen du peuplement tout en conservant un potentiel de production élevé qui permette de préserver le couvert forestier et de revenir en coupe avec des rotations courtes ; - exploitation en coupe rase (hors pente et bord de crique) permettant de concentrer le prélèvement. Ce scénario ne peut s'envisager que dans le cas où toutes les essences sont valorisées. II impose d'étudier sérieusement les questions de la préservation des sols et de la biodiversité et présente de nombreuses limites y compris d'image. 
Il n'est donc pas envisagé concrètement mais il est ici étudié comme scénario témoin pour évaluer la productivité théorique de la forêt et calculer les coûts de mobilisation du bois.

Pour le scénario « exploitation intensive ", il s'agira de prélever toutes les tiges exploitables de 40 à $80 \mathrm{~cm}$ de diamètre, soit environ 40 tiges à l'hectare, ce qui représente $100 \mathrm{~m}^{3} / \mathrm{ha}$. Un tel scénario se rapproche du traitement 3 appliqué sur le dispositif de recherche de Paracou avec une réduction de l'ordre de $30 \%$ de la surface terrière. L'expérience met en évidence une production nette de 5 à $6 \mathrm{~m}^{3} /$ ha/an durant les vingt années après l'exploitation. Les bois de 25 à $40 \mathrm{~cm}$ de diamètre ont une densité dans le peuplement et un accroissement annuel moyen de $0,4 \mathrm{~cm}$ compatibles avec le maintien du stock exploitable dans la catégorie $35-50 \mathrm{~cm}$ de diamètre.

Les travaux ont montré que le volume de bois sur pied se stabilise après vingt-cinq ans aux environs de $200 \mathrm{~m}^{3}$ de grumes par hectare. Ce scénario nécessite un contrôle rigoureux du débardage, car la fréquence de passage des engins risque de déstructurer des sols déjà fragiles, comme le montrent des études réalisées en Amazonie (Mc NABB et al., 1997).

Le calcul du volume de bois disponible pour une forêt de 10000 ha, exploitée tous les vingt-cinq ans par parcelles de 200 ha, dont $50 \%$ de la surface est exploitable 7 , aboutit à une production annuelle de $20000 \mathrm{~m}^{3}$.

Dans le cas du scénario « coupe rase ", la totalité du peuplement serait exploitée. L'expérience d'Arbocel ${ }^{8}$ montre que, vingt-sept ans après, le peuplement reconstitué totalise seule- ment $130 \mathrm{~m}^{3}$ de grumes à l'hectare, formé à $95 \%$ de tiges de 10 à $40 \mathrm{~cm}$ de diamètre. Un minimum de quarantecinq ans serait nécessaire pour garantir une reconstitution suffisante pour une nouvelle exploitation. Si l'étude de l'érosion des sols (SARRAILH, 1990) conclut sur l'absence de risque majeur vis-à-vis de la perte de fertilité du sol et de la qualité des eaux, elle émet cependant des réserves dues à la faible superficie des bassins versants étudiés. Un tel scénario impliquerait une extraction maximum de la biomasse, houppiers compris, afin de limiter le risque d'incendie. Or, l'utilisation des houppiers pose un grand nombre de problèmes techniques vus précédemment.

En considérant une production effective de $260 \mathrm{~m}^{3} / \mathrm{h}$ a et une rotation de quarante-cinq ans, 80 ha sont suffisants pour une production annuelle équivalente à la précédente. Au final, le scénario aboutirait à une productivité similaire (hors houppiers) à celle de l'exploitation intensive, comme l'illustre le tableau II.

Ce scénario ne permettrait donc pas réellement d'intensifier la production de bois énergie. De plus, l'ensemble des problèmes environnementaux posés souligne l'aspect irrationnel qu'il y a à l'envisager. Les estimations qui suivent correspondent donc au scénario " exploitation intensive ». Le volume total mobilisable pour le gisement en exploitation forestière durable à vocation énergétique a été estimé à $125000 \mathrm{~m}^{3}$ par an en 2007, mais des surfaces supplémentaires pourraient être identifiées pour répondre à la demande future.

\section{Bilan sur les deux gisements de biomasse forestière pour l'énergie}

Comparativement à l'exploitation mixte, le principal intérêt de l'exploitation en forêt à vocation énergétique est de limiter la surface mise en exploitation, en plus de la mise en réserve obligatoire de zones non exploitées pour préserver la biodiversité et la fonctionnalité des écosystèmes. L'exploitation de la forêt à ces fins devrait ainsi faciliter l'organisation des chantiers, limiter les investissements, permettre de réduire le transport du bois, et garantir une grande régularité dans l'approvisionnement. La forêt s'en trouve par contre totalement transformée dans sa composition et son fonctionnement.

L'obstacle majeur reste le peu de connaissances sur les impacts à long terme d'une mécanisation intensive sur des sols tropicaux sensibles.

Au plan environnemental, pour les deux gisements, le niveau modéré de prélèvement et le maintien en forêt des houppiers et tiges de faible diamètre minimisent l'exportation de matières minérales et de matière organique, ce qui devrait maintenir les caractéristiques des sols pour ces éléments. Des recherches sont cependant à mener pour confirmer cette hypothèse.

\footnotetext{
7 Après exclusion des bas-fonds et fortes pentes (> $40 \%$ ).

${ }^{8}$ Arbocel (Arbre et cellulose) : expérience de coupe rase de bois, composante de l'étude Ecerex (ECologie, ERosion, EXpérimentation), qui a consisté en l'étude de la mise en valeur de l'écosystème forestier guyanais après déboisement dans les années 1980.
}

\begin{abstract}
Tableau II.
Calcul de référence pour l'estimation des quantités mobilisables annuellement sur un massif aménagé de 10000 ha consacré uniquement à la production de bois énergie (source : Onf Guyane).
\end{abstract}

Scénario

Coupe forte

Coupe rase

(sans les houppiers)
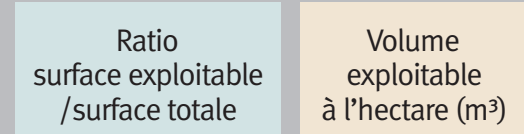

0,5

0,4

100

260
Rotation nécessaire entre deux coupes (ans)
Surface annuelle exploitable sur 10000 ha

25

50
Production annuelle sur 10000 ha $\left(\mathrm{m}^{3}\right)$ 


\section{Itinéraire technique et coûts de mobilisation de la biomasse}

Les itinéraires techniques des scénarios d'exploitation des deux gisements de bois énergie en gestion durable des forêts ont été élaborés à partir d'expériences techniques et de données de terrain.

Pour l'exploitation forestière mixte, l'expertise s'est appuyée sur les données actuelles de l'exploitation de bois œuvre, afin de définir des modalités d'exploitation du bois énergie et d'extrapoler les coûts des unités d'œuvre. Ainsi, l'abattage et le débardage ont été prévus selon des modalités classiques : abattage manuel à la scie à chaîne et débardage à la traîne. Les coûts prévisionnels sont de 4 euros par mètre cube $\left(€ / \mathrm{m}^{3}\right)$ pour l'abattage avec un rendement de 75 à 80 mètres cubes par jour $\left(\mathrm{m}^{3} / \mathrm{j}\right)$ à deux opérateurs et de $17 € / \mathrm{m}^{3}$ pour le débardage avec un rendement machine de $120 \mathrm{~m}^{3} / \mathrm{j}$ à deux opérateurs.
Les charges à prévoir en termes de gestion forestière sont une usure des pistes accentuée compte tenu du triplement du prélèvement, mais aussi un contrôle des coupes plus lourd et une intégration de l'objectif dans les aménagements. À ce titre, un prix du bois énergie de $3 € / \mathrm{m}^{3}$ sur pied semble être un niveau raisonnable de rémunération. Au total, le bois revient à $24 € / \mathrm{m}^{3}$, soit vingt euros par tonne, rendu bord de piste.

Quant à l'exploitation en forêt pour le bois énergie, l'itinéraire technique a été établi par référence à la filière du bois d'œuvre actuelle. Des coefficients de réduction des coûts ont été utilisés pour tenir compte de l'intensification des prélèvements et de la plus grande productivité horaire des chantiers. Le coût d'abattage a été défini à $3,5 € / \mathrm{m}^{3}$, sur la base de $110 \mathrm{~m}^{3} / \mathrm{j}$ à deux opérateurs, et $15 € / \mathrm{m}^{3}$ pour le débardage, sur la base de $200 \mathrm{~m}^{3} / \mathrm{j}$ à deux opérateurs.
La desserte des parcelles nécessite un investissement supplémentaire en infrastructure évalué entre trois et quatre kilomètres pour 1000 ha avec un coût moyen de vingt-six mille euros par kilomètre de piste. La prise en charge de l'aménagement de ces massifs, amorti sur les vingt-cinq ans de la durée de rotation, justifie un prix de vente du bois énergie de l'ordre de $5 € / \mathrm{m}^{3}$. Le coût du bois s'établit ainsi à $23,5 € / \mathrm{m}^{3}$, soit $19,6 € / t$ en bord de piste.

Le tableau III présente le coût de collecte, soit le transport jusqu'à la centrale et la mise en plaquettes du bois. Les données pour le bois de déforestation lors de la création des pistes forestières sont indiquées car cette ressource pourrait utilement participer à l'approvisionnement de la centrale dans l'étude de cas ci-après. L'évaluation de ce gisement a été présentée dans l'article publié dans les actes de la conférence WasteEng Africa 2009 (PINTA, GIRARD, 2009).

Tableau III.

Coût du bois énergie, mis en plaquettes, rendu au silo de stockage (PINTA et al., 2007).

\begin{tabular}{l|ccc}
$\begin{array}{l}\text { Gisement origine } \rightarrow \\
\text { Coût des opérations }(€ / t)\end{array}$ & $\begin{array}{l}\text { Exploitation mixte (bois } \\
\text { d'œuvre et bois énergie) }\end{array}$ & $\begin{array}{c}\text { Exploitation forêt } \\
\text { bois énergie }\end{array}$ & $\begin{array}{c}\text { Création des } \\
\text { pistes en forêt }\end{array}$ \\
$\begin{array}{l}\text { Achat de la biomasse } \\
\begin{array}{l}\text { Coût de la récolte et mise } \\
\text { en dépôt bord de piste }\end{array}\end{array}$ & 3 & 5 & 0 \\
\hline $\begin{array}{l}\text { Transport sur } 20 \mathrm{~km} \text { et mise } \\
\text { en plaquettes forestières }\end{array}$ & 17 & 14,6 & 10,7 \\
\hline Total & 12,4 & 12,4 & 12,4 \\
\end{tabular}

Tableau IV.

Volumes disponibles par type de gisement dans la zone d'étude à Régina (PINTA et al., 2007).

\begin{tabular}{l}
$\begin{array}{l}\text { Éloignement } \\
\text { centrale }(\mathrm{km})\end{array}$ \\
$0-12,5$ \\
$12,5-25$ \\
\hline Total Régina \\
\hline
\end{tabular}

$\begin{aligned} & \text { Forêt bois énergie } \\ & + \text { bois d'œuvre }\end{aligned}$
481900
140300
622200
Zone agricole
création pistes

$$
0
$$

95700 95700

\begin{tabular}{|} 
Déforestation \\
0 \\
457600 \\
457600 \\
\hline
\end{tabular}

\section{Total $\left(\mathrm{m}^{3}\right)$}

481900

663600

1145500 


\section{Étude de cas d'une centrale de cinq mégawatts à Régina}

Dans la zone de Régina, la biomasse énergie disponible est abondante (tableau IV) mais la distance à un poste source du réseau Edf est aujourd'hui élevée. L'étude anticipe l'existence future du réseau entre Régina et Saint-Georges-de-l'Oyapock. La localisation de la zone d'exploitation retenue est illustrée en figure 2 .

\section{Provenance et volume prévisionnel de la biomasse}

Le tableau IV fournit une indication des volumes par gisement potentiel en fonction de leur éloignement respectif de la centrale selon des cercles concentriques de douze et demi à vingtcinq kilomètres $(\mathrm{km})$ de rayon.

L'approvisionnement de la centrale pourra être assuré à partir de bois de récupération sur la création des pistes pour $46 \%$ et de bois issus de l'exploitation mixte pour $54 \%$ sur vingt ans, soit un million de tonnes. Les bois de défriche agricole dans la zone seront mobilisés en substitution ou complément, selon le besoin au moment de leur disponibilité.

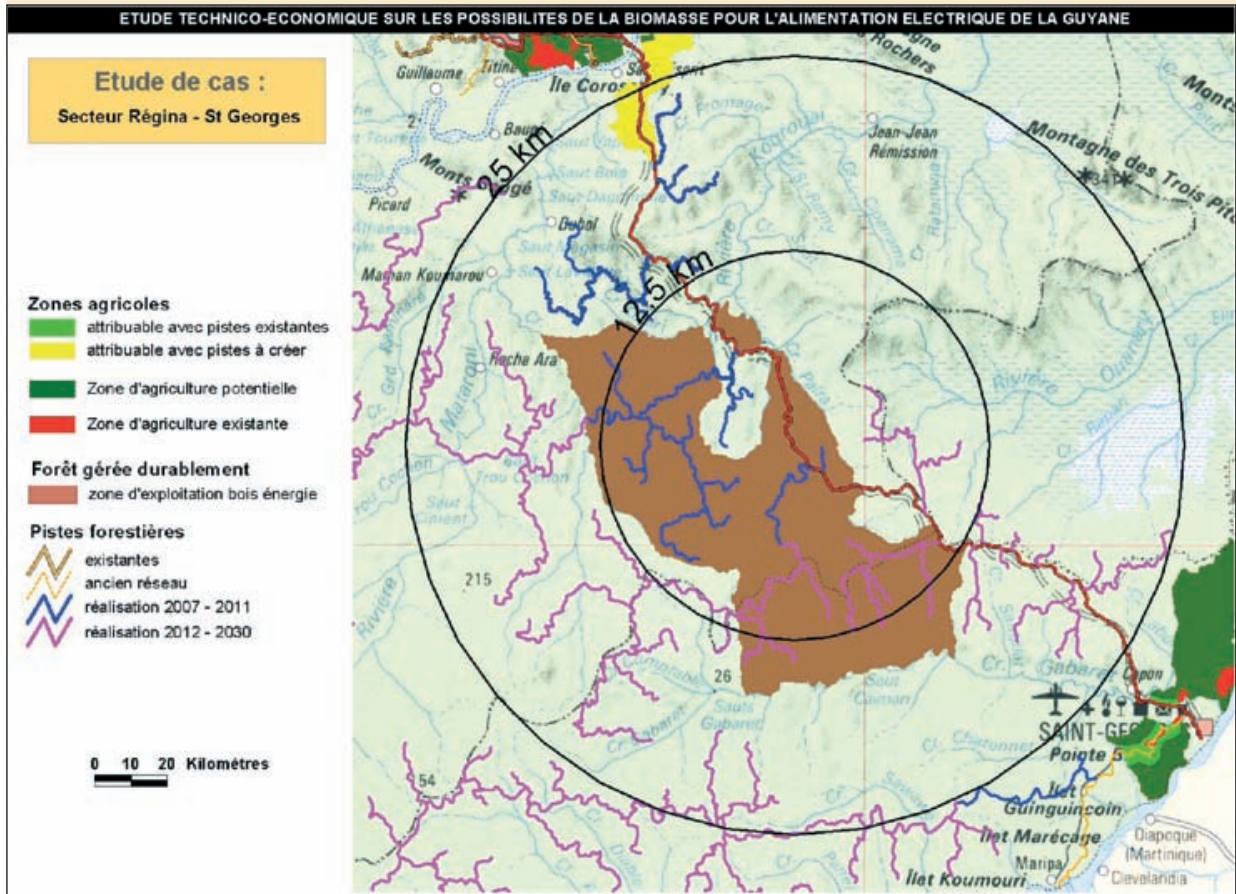

Figure 2.

Localisation de la zone d'exploitation envisagée à proximité de Régina.

\section{Analyse économique et financière}

La production annuelle nette d'électricité de la centrale étudiée à Régina est calculée à trente-cinq mille sept cents kilowattheures (kWh). Les données de base et les principaux résultats de l'analyse économique et financière sont présentés dans le tableau V. Le coût du transport retenu en 2007 à $0,22 € / \mathrm{t} / \mathrm{km}$ sur une distance moyenne de $20 \mathrm{~km}$ fait ressortir un coût moyen de $4,4 € / t$.

Tableau V.

Synthèse des principales hypothèses et des résultats de l’étude de cas de Régina (PıNTA et al., 2007).

Montant des investissements $(\mathrm{k} €)$

Prix d'achat biomasse forestière $(€ / t)$

Prix moyen de revient plaquette forestière entrée centrale $(€ / t)$

Prix de vente (€/MWh électrique)

Résultat du projet

Taux interne de rentabilité (\%)

Temps de retour (années)

Prix de revient actualisé à $8 \%$ ( $€ / M W h)$

\begin{tabular}{|c|c|}
\hline $\begin{array}{l}\text { Hypothèse } \\
\text { référence }\end{array}$ & $\begin{array}{l}\text { Hausse du prix de } \\
\text { vente de l'électricité }\end{array}$ \\
\hline 16000 & 16000 \\
\hline 23,34 & 23,34 \\
\hline 31,34 & 31,34 \\
\hline 105 & 160 \\
\hline 0 & 19 \\
\hline$>20$ & 5 \\
\hline 137 & 137 \\
\hline
\end{tabular}

Hausse du coût de la biomasse

16000

26,34

34,34

160

16

7

142
Hausse des investissements

18400

23,34

31,34

160 


\section{Conclusion}

Le prix de revient varie entre 137 et $155 € / M W h$ électrique, niveau intéressant au regard de ce que sont les coûts de production à partir d'énergie fossile en Guyane française, proches de $200 € / M W h$. Contrairement aux options qui permettaient de valoriser principalement des bois de défriche agricole (PINTA, GIRARD, 2009), un prix de rachat de $105 € /$ MWh ne permet pas une rentabilité du projet. En tenant compte de l'origine forestière du bois, Edf pourrait envisager aujourd'hui un prix de rachat de $160 € / M W h$, qui permettrait la faisabilité du projet. De plus, l'étude de sensibilité montre que cette option est très sensible au prix du bois et au coût d'investissement. Même si cette solution énergétique présente des impacts socioéconomiques positifs pour la région (Anonyme, 2003), elle ne pourrait pas s'envisager sans subvention et surtout sans une recherche d'accompagnement pour définir les conditions techniques optimales de mobilisation du bois énergie.

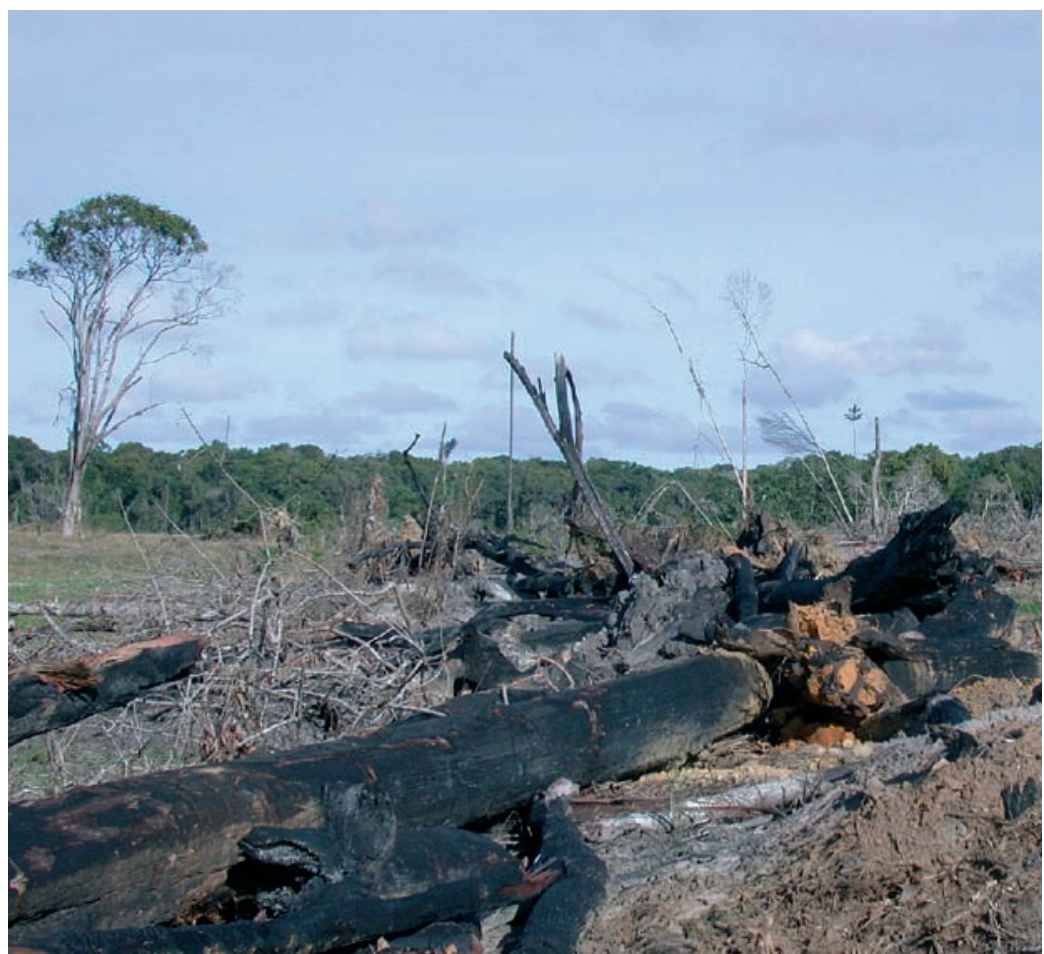

Photo 5.

Bois énergie récupérable lors de la conversion en parcelle agricole (ici en pâturage en Guyane française).

Photo F. Pinta.
L’approvisionnement de centrales énergétiques à partir de forêt naturelle en exploitation durable apparaît économiquement plausible dans le contexte de la Guyane française. Ici comme dans la plupart des pays forestiers tropicaux, l'exploitation durable des forêts pour l'énergie correspond à une logique de développement économique local. Cette option mériterait d'être approfondie notamment dans d'autres contextes économiques, l'ensemble des coûts unitaires et particulièrement de main-d'œuvre étant très élevés en Guyane française.

Une exploitation durable et maximalisée des forêts naturelles représente l'un des leviers pour leur préservation par rapport à une logique de défriche à des fins agricoles. Comme suggéré par les instances internationales telles que la Fao, l'intégration de la production d'énergie dans les opérations forestières industrielles permet d'améliorer la gestion forestière d'une façon compétitive et de contribuer à l'atténuation du changement climatique.
En outre, l'exploitation forestière durable mixte s'inscrit clairement dans la logique d'aménagement durable des forêts tropicales. La production simultanée de bois énergie et de bois d'œuvre permettra, d'une part, de diversifier la gamme d'essences exploitées et, d'autre part, de stimuler la reconstitution de la forêt en offrant un potentiel d'amélioration de la production de bois d'œuvre.

Le manque de connaissances et d'expérience sur ce type d'exploitation en forêt naturelle tropicale humide se traduit par un besoin de recherches nouvelles. L'objectif est d'établir les méthodologies d'aménagement durable incluant le bois énergie et les modalités pratiques de mise en œuvre, tant en forêt à vocation énergétique qu'en forêt à vocation mixte.

Cette logique est déjà en cours en forêt tropicale sèche et largement pratiquée, avec certes des résultats parfois mitigés. Les forêts y sont pourtant très fragiles et subissent les impacts répétés des incendies, de la sécheresse, ainsi qu'une forte pression anthropique caractérisée par une exploitation essentiellement artisanale, peu formée et ne disposant que de peu de moyens techniques et financiers.

Il est aujourd'hui temps d'étudier comment on peut réaliser une exploitation durable à des fins énergétiques dans des forêts présentant un potentiel de production bien supérieur, sachant que les acteurs économiques ont démontré, dans bien des régions, leur capacité à faire évoluer leurs pratiques pour une gestion durable des forêts tropicales humides.

\section{Remerciements}

Les auteurs remercient les personnes avec lesquelles ils ont collaboré pour réaliser ce travail et particulièrement Pierre Courtiade de l'Ademe Guyane qui a cofinancé l'étude sur laquelle s'appuie ce travail, Florent Ingrassia, Stéphane Guitet et Ambroise Graffin de l'Office national des forêts. 


\section{Références bibliographiques}

ANONYME, 2003. Plan énergétique régional, tome 2 : propositions d'actions. ICE DEBAT, Région Guyane, 61 p. ANONYME, 2007. Programme régional de mise en valeur forestière. Office national des forêts, Guyane, 13 p.

BERTRAND A., MONTAGNE P., 2009. Stratégie énergie domestique et gestion des ressources forestières au Niger et au Mali : gestion, domanialité, fiscalité et contrôle forestier. Bois et Forêts des Tropiques, 301 : 85-99.

DEMENOIS J., FUHR M., GOURLETFLEURY S., JOURGET J.-G., 2003. Sylviculture en forêt tropicale humide guyanaise. Revue forestière française, 55 (numéro spécial Connaissance et gestion de la forêt guyanaise) : 273-290.

FALCH F., PINTA F., 2007. Détermination du taux d'humidité dans le bois sur pied chez quelques espèces de la forêt guyanaise. Quelles conséquences pour une valorisation en bois énergie? Rapport de stage, licence Protection de l'environnement, Université Antilles-Guyane, Cirad, France, 40 p.

FAO, 2008. Les forêts et l'énergie. Questions principales. Rome, Italie, Fao, Étude Forêts nº 154, 82 p.

GIRARD P., VERGNET L.-F., VERNAY M., 1997. Contribution de la biomasse au bilan énergétique de la Guyane : bilan et perspectives. Prme, programme européen Altener, CiradForêt, 3 tomes, $107 \mathrm{p}$.

GOURLET-FLEURY S., BLANC L., PICARD N., SIST P., DICK J., NASI R., SWAINE M. D., FORNI E., 2005. Grouping species for predicting mixed tropical forest dynamics : Looking for a strategy. Annals of Forest Science, 62 (8) : 785-796.

GUITET S., BLANC L., TROMBE P.-J., LEHALLIER B., 2009. Traitements sylvicoles en forêt tropicale guyanaise : bilan de dix ans d'expérimentations. Bois et Forêts des Tropiques, 301 : 7-19.
ICHAOU A., ROULETTE G., 2006. Perspective de durabilité des modèles de gestion locale des formations forestières développés au Niger. In : Bertrand A., Montagne P., Karsenty A. (éd.). L'État et la gestion locale durable des forêts en Afrique francophone et à Madagascar. Paris, France, L'Harmattan, p. 259-267.

MC NABB K. L., MILLER M. S., LOCKABY B. G., STOKES B. J., CLAWSON R. G., STANTURF J. A., SILVA J. N. M., 1997. Selection harvests in Amazonian rainforests : long-term impacts on soil properties. Forest Ecology and Management, 93 : 153-160.

PINTA F., GIRARD P., 2009. Analyse de la disponibilité en biomasse pour la production d'électricité en Guyane française : opportunités et contraintes pour les déchets de bois mobilisables. In : Actes de la conférence WasteEng Africa, 9-11 juillet 2009, Ouagadougou, Burkina Faso.

PINTA F., GIRARD P., GRAFFIN A., INGRASSIA F., 2007. Étude technicoéconomique sur les possibilités de la biomasse pour l'alimentation électrique de la Guyane française. Cirad, Onf, Ademe Guyane, 110 p.

SARRAILH J.-M., 1990. Mise en valeur de l'écosystème forestier guyanais. Opération Ecerex. Nogent-sur-Marne, France, Cirad-Ctft, 273 p. 